\title{
EFEITOS DA CINESIOTERAPIA NAS DISFUNÇÕES SEXUAIS DA MULHER NA PÓS-MENOPAUSA
}

\section{REVISÃO INTEGRATIVA}

LUCENA, Clemilda Braga de ${ }^{1}$

FREIRE, Lorena Lacerda ${ }^{2}$

LUCENA, Clemilda Braga de. FREIRE, Lorena Lacerda. Efeitos da cinesioterapia nas disfunções sexuais da mulher na pós-menopausa. Revista Científica Multidisciplinar Núcleo do Conhecimento. Ano 05, Ed. 08, Vol. 05, pp. 106-118. Agosto de 2020. ISSN: 2448-0959, Link de acesso: https://www.nucleodoconhecimento.com.br/saude/efeitos-da-cinesioterapia

\section{RESUMO}

O período pós-menopausa corresponde as alterações na fase denominada de terceira idade; caracterizando uma interrupção dos níveis hormonais e consequentemente irregularidades nos ciclos menstruais, nessa relação há sintomas como diminuição do desejo sexual, encurtamento da vagina, fraqueza dos músculos do assoalho pélvico, falta de lubrificação e alterações na microbiota vaginal. A maioria das mulheres não compreendem sobre a importância da atuação da Fisioterapia nas disfunções sexuais acometidas na pós-menopausa, consequentemente dão preferência a uma vida sexual inativa. O objetivo desse estudo é descrever os efeitos da cinesioterapia nas disfunções sexuais da mulher na pós-menopausa. Trata-se de uma Revisão Integrativa de Literatura; o levantamento bibliográfico foi realizado no período de janeiro a junho de 2019, por meio das bases de dados SCIELO, LILACS e MEDLINE, nos idiomas português, inglês e espanhol, a busca se deu pelos descritores: Menopausa. Fisioterapia; Assoalho Pélvico; Incontinência urinária; utilizando o

${ }^{1}$ Graduação em Fisioterapia.

${ }^{2}$ Graduada em Fisioterapia e Pós-Graduada em Fisioterapia Pélvica. 
operador booleano AND para pesquisa. Os resultados apresentaram o acordo sobre a intervenção de exercícios para o fortalecimento da musculatura pélvica através da cinesioterapia, técnica terapêutica eficaz no tratamento das disfunções sexuais por consequências expostas. Conclui-se que, apesar da limitação de artigos, a cinesioterapia como recurso fisioterapêutico proporciona significativa melhora na qualidade de vida de mulheres na pós-menopausa, além de tornar-se compreensível às alterações fisiológicas neste período.

Palavras-chave: Menopausa, fisioterapia, assoalho pélvico, incontinência urinária.

\section{INTRODUÇÃO}

Desde sempre as mulheres vem sofrendo um processo de transição na vida adulta, o climatério que resulta na passagem da última menstruação para a menopausa (SILVA et al., 2018). Nesse processo acontece a interrupção dos níveis de hormônios femininos e se manifestam sintomas como irregularidades nos ciclos menstruais, sensações de calor, risco de doenças cardiovasculares, alteração de humor e principalmente diminuição no desejo sexual como consequência das disfunções urogenitais (FONSECA et al., 2018; LOPES, 2018).

Atualmente, a menopausa é considerada um problema de saúde pública, em relação ás consequências de sua prevalência (OLIVEIRA et al., 2018). A menopausa passou a ser um grande desafio em mulheres, principalmente quando ela surge antes do período esperado, podendo causar problemas no meio social, alterações nos aspectos físicos e emocionais, além de ser associado a diminuição da libido, afetando assim a sexualidade do casal no lar, pois o desconforto ou incompreensão sobre essas alterações orgânicas e não orgânicas levam as mulheres ao abandono com o sexo (ALMEIDA et al., 2018).

Na pós-menopausa o epitélio vaginal torna se mais delgado e seco, causando assim encurtamento da vagina, em caso de atrofia severa a superfície da vagina pode sangrar com facilidade e apresentar estenose. A mucosa pode ficar menos elástica, com alterações na microbiota vaginal e diminuição do fluxo sanguíneo, além de 
sintomas sexuais, como falta de lubrificação e dificuldades durante relações sexuais (KAMILOS et al, 2017).

Outros problemas como incontinência urinária, fraqueza dos músculos do assoalho pélvico, ressecamento da mucosa vaginal e deficiência nos hormônios estrogênio e progesterona estão presentes na vida da mulher na pós-menopausa. Levando a necessidade de uma intervenção fisioterapêutica tanto na prevenção, como no tratamento desses sintomas, atuando no fortalecimento e resistência dos músculos envolvidos, minimizando a frouxidão dos ligamentos pélvicos e manutenção do desempenho sexual (JESUS et al., 2018; CREMA et al., 2017).

Acompanhar a saúde da mulher climatérica e conhecer suas condições de saúde são medidas fundamentais para adoção de estratégias preventivas e de promoção de saúde, evitando morbidades e permitindo uma melhor qualidade de vida nessa fase (SILVA et al.,2018).

Nesse sentido, surgiu o seguinte questionamento: Quais intervenções fisioterapêuticas atuam nas disfunções sexuais ocasionadas em mulheres na pósmenopausa?

Dentro desse contexto, a pesquisa se propõe a contribuir com mais conhecimento acerca do assunto, para possibilidade de mulheres passarem pela pós-menopausa de um modo mais confortável, pois são muitos sintomas presentes na vida dessas mulheres e a fisioterapia acompanhando esse processo logo no inicio irá atuar tanto de forma preventiva como na minimização da sintomatologia, ressaltando a necessidade de estimular e fortalecer a musculatura perineal para que assim possa ser evitadas outras disfunções do assoalho pélvico.

\section{AVALIAÇÃO DA MUSCULATURA PÉLVICA NA PÓS-MENOPAUSA}

A avaliação consiste em uma anamnese do paciente, seguida de um exame físico com o toque bi digital e aparelho de biofeedback manométrico que irão estabelecer o grau de força da musculatura pélvica por meio do método PERFECT. O exame é feito 
com a paciente deitada em decúbito dorsal com joelhos fletidos a $45^{\circ}$, o fisioterapeuta irá introduzir os dedos indicador e médio com o uso de gel lubrificante e luvas, posteriormente é solicitado que a paciente contraia a musculatura em torno dos dedos, a capacidade de força de contração dessa musculatura é graduada de 0 a 5 de acordo com escala de PERFECT (RIBEIRO et al., 2012).

O biofeedback é considerado um aparelho que mede, avalia e trata as disfunções neuromusculares, sendo muito importante na avaliação dos músculos do assoalho pélvico, pois irá monitorar o tônus em repouso dessa musculatura, a força e a sustentação, além de conscientizar o paciente de seu corpo e suas funções através de estímulos táteis, visuais, auditivos e elétricos (TOME et al., 2015).

\subsection{ALTERAÇÕES BIOMECÂNICAS NA PÓS-MENOPAUSA}

A menopausa ocorre em torno de 45 a 50 anos de idade, e é um episódio pontual durante o climatério e se caracteriza pela finalização permanente da menstruação após um período de 12 meses consecutivos. Ondas de calor, secura vaginal, sudorese, dificuldades de excitação e alterações no funcionamento sexual são os principais sintomas prevalentes nesse período, podendo acontecer de forma espontânea ou em decorrência de intervenções médicas (CREMA et al., 2017).

Os estrogênios são os hormônios sexuais que envolvem o aparelho genital ou trato urinário inferior, e na pós-menopausa acontece muitos sinais e sintomas relacionados diretamente com a diminuição dos níveis desse hormônio, apresentando distúrbios da geniturinária na pós-menopausa. As mulheres podem sofrer com a síndrome geniturinária por mais de um terço da sua vida, e precisa ser tratada com opções além de reposições hormonais, para que assim o tratamento da atrofia vaginal melhore a qualidade de vida dessas mulheres (SANTOS et al., 2017).

Existe uma crescente incidência de Doença Arterial Coronariana (DAC) na população do sexo feminino, principalmente no período do climatério, e essas alterações estão relacionadas as mudanças hormonais, circulatórias e sanguíneas que ocorrem na mulher, a redução de morbidade e mortalidade é a identificação antecipada dos 
fatores de risco cardiovasculares em mulheres climatéricas (MELO et al., 2017; FARIAS et al., 2017).

A perda de continência urinaria em mulheres na pós-menopausa é um problema tanto familiar como social, pois acontece um desconforto e constrangimento de perder urina com pouco esforço, ficarem molhadas grande parte do tempo e controlar a ingestão de líquidos. Essas mulheres acabam que se isolando com medo de estarem em um espaço público e perderem urina antes de chegarem no banheiro e de apresentarem odor de urina, tais problemas causam problemas psicológico e emocionais (BERLEZI et al., 2009).

Outro acontecimento na pós-menopausa são as disfunções sexuais, onde acontece um menor interesse ou desejo de começar uma atividade, ou seja, diminuição da excitação ou da capacidade de atingir o orgasmo durante relações sexuais, podendo causar problemas complexos e com muitas etiologias (LOMÔNACO et al., 2015).

Após a menopausa, muitas mulheres passam a apresentar distúrbios do sono, e no caso das que já apresentavam dificuldades para dormir, as queixas tornam-se mais prevalentes. Um sono ineficaz pode comprometer suas tarefas diárias, seu bem-estar mental e físico durante o dia e, consequentemente, sua qualidade de vida, e isso se acontece devido mudanças hormonais que ocorrem nessa fase (KWEON et al., 2017).

Considerando que mulheres na pós-menopausa apresentam alterações na competência funcional, com uma diminuição na resistência muscular e na flexibilidade, com um parâmetro de classificação funcional distinto em relação às mulheres que ainda não atingiram esse estágio (NEVES et al., 2015)

As mulheres associam o processo de menopausa como uma forma particular de envelhecimento, focando no fim da vida reprodutiva, entendem que a menopausa é um marco que deve ser enfrentado na última etapa da vida. E essa percepção acaba que resultando em sérios problemas psicológicos com essas mulheres (ARAYA et al., 2017). 


\subsection{DISFUNÇÕES SEXUAIS NA PÓS MENOPAUSA}

A sexualidade é um componente inerente à vida de todos os seres humanos, que se inicia antes mesmo do nascimento e só se encerra com a morte, além de influenciar na forma de como se posicionar no mundo. A sexualidade se dá sobre os aspectos biopsicossociais de cada indivíduo, abrangendo seu potencial biológico, suas emoções, sentimentos, crenças e concepções desenvolvidas, ampliadas e modificadas durante todo o processo de socialização (GALATI et al., 2014).

O papel central da sexualidade na saúde global dos indivíduos justifica o estimulo a educação sexual dos profissionais da saúde, pois a promoção de saúde sexual depende da qualidade da formação profissional em todos os aspectos em envolvem a sexualidade. Essas entidades afirmam que a promoção da saúde sexual envolve a implementação de ações que contemplem toda a abrangência da sexualidade humana, para que assim todas as pessoas que estiverem passando por problemas sexuais procurem um profissional de saúde (RUFINO et al., 2013).

A disfunção sexual feminina é caracterizada quando fatores relacionados a sexualidade são prejudicados, caracterizada por uma perturbação clinicamente significativa na capacidade de uma pessoa responder ou experimentar prazer sexual, e seu impacto pode causar aspectos na qualidade de vida, como dor, estado geral, aspectos emocionais, capacidade e saúde mental (BARRETO et al., 2018).

As disfunções sexuais na pós-menopausa estão relacionadas ao desconforto que acontece com a mulher durante a relação sexual, entre as principais queixas estão o posicionamento durante $o$ ato sexual, a falta da libido, o medo de exacerbação da dor, a depressão, os problemas de relacionamentos e confidências. Acontece uma diminuição na frequência com que o orgasmo acontece, satisfação sexual e excitação são diminuídas, e isso pode provocar dispaureunia por diminuição do fluxo sanguíneo para a vagina (SANTOS et al., 2015).

Apesar das disfunções serem bem conhecidas, muitas mulheres não apresenta a queixa dos problemas sexuais que estão passando aos médicos por vergonha e 
constrangimento da investigação e do tratamento, e isso acaba que interferindo na qualidade de vida dessas mulheres, pois esse diagnóstico é muito relevante em questão de saúde geral, pois se tratando logo no início dos sintomas, irá ser mais difícil apresentar problemas secundários (HOLANDA et al., 2014).

$\mathrm{Na}$ sexualidade vem sendo identificada por distúrbios ou disfunções principalmente em mulheres, podendo ser de causa física e do tipo não orgânica, sabendo-se que existem várias causas que podem causar esses distúrbios. O ideal é que a sexualidade feminina acorde de todos os tabus e restrições que sofreu há décadas e que essas mulheres possam mostrar seus pensamentos, fantasias e desejos, para se tornarem uma parte integrante de mulheres compreendidas como pessoa e no relacionamento como casal (BAQUEDANO, 2017).

\subsection{CINESIOTERAPIA NA PÓS-MENOPAUSA}

A cinesioterapia é um dos tratamentos mais eficazes para fortalecer o assoalho pélvico, podendo ser associado ao cones vaginal, que tem como objetivo o ganho de força muscular, melhorando a sintomatologia e diminuindo a perda urinaria, além de ser composto por um material de qualidade e possuir na sua extremidade um fio de nylon para facilitar a sua retirada (MENDES et al., 2017; SILVA, 2018).

Outro tratamento que vêm sendo muito utilizado nas condutas para fortalecimento da musculatura pélvica são os exercícios de kegel, onde se baseiam em contrações voluntarias da musculatura do assoalho pélvico. Podendo ser realizado em várias posições e com auxílio de bolas suíças. Esses exercícios são de grande importância para incontinência urinaria e vaginismo, além de melhorar o prazer sexual devido ao aumento da circulação sanguínea (SOUSA et al., 2011).

Para aumentar a força perineal e ativar o musculo transverso do abdome é a ginastica hipopressiva, que tem como objetivo tonificar o assoalho pélvico e melhorar a circulação, e consequentemente melhorar o desemprenho sexual que geralmente acontece na pós-menopausa, além de combater a perca de urina e de fezes devido 
ao fortalecimento da musculatura e dos ligamentos que envolvem essas estruturas (VALENTE et al., 2015).

O método de execução física pilates é um ótimo recurso para o tratamento de mulheres na pós-menopausa, pois nesse período acontece uma baixa taxa de hormônios, e com isso um aumento significativo no risco de quedas, sendo assim o pilates irá auxilia na força, flexibilidade e resistência dessas mulheres, fazendo com que haja uma diminuição de fraturas nessa fase e garantindo um maior equilíbrio motor (SOUZA et al., 2013).

A prática regular de exercícios terapêuticos na piscina estimula a secreção de endorfinas, causando sensação de bem estar, o que irá beneficiar nos sintomas de mulheres na pós-menopausa, proporcionando diminuição de ansiedade e alivio nas ondas de calor, e tendem a manifestar melhoria no humor. Mulheres que fazem exercícios regulares possuem uma maior preservação de massa muscular e uma maior capacidade respiratória, sem falar que exercícios tendem a normatização da pressão arterial (AGRA et al., 2013).

Algumas mulheres na pós-menopausa acabam evidenciando a alta prevalência de níveis elevados de pressão arterial, e como medidas não farmacológicas envolvendo mudanças no estilo de vida, a prática regular de exercícios físicos aeróbicos vem sendo indicada por profissionais da saúde como um meio efetivo para o controle dos níveis elevados de PA. (LIMA et al., 2011).

A fisioterapia pélvica pode de fato contribuir na resolução de distúrbios e na melhoria de qualidade de vida de mulheres que passam por distúrbios no assoalho pélvico. No decorrer dos anos, a atuação da fisioterapia na área de ginecologia, obstetrícia e urologia vem ganhando espaço por participar das várias fases da vida, especialmente, da mulher. Cuidando do seu bem-estar e promovendo sua saúde, contribuindo para a manutenção da autoestima e qualidade de vida, e cada vez mais os médicos fazem encaminhamento para os fisioterapeutas que atuam nessa área (STEIN et al., 2018). 


\section{CONCLUSÃO}

Espera-se que essa revisão integrativa auxilie futuros pesquisadores no tema ao abordar a importância da fisioterapia no período pós-menopausa, apresentando os melhores recursos que a cinesioterapia pode oferecer para as mulheres, compreendendo as alterações que acontecem fisiologicamente nesse período.

Os autores evidenciaram benefícios de todos os recursos da cinesioterapia utilizados no fortalecimento dos músculos do assoalho pélvico, especificando a melhora na qualidade de vida por contribuir para a reabilitação das disfunções ocorridas no período do climatério.

Este estudo confirmou a carência de informações sobre a área da Fisioterapia Pélvica e os benefícios da cinesioterapia nas disfunções sexuais pós-menopausa. Faz-se necessária a atenção ao desenvolvimento de práticas que contribuam na saúde de mulheres no período pós-menopausa, visto sua relevância preventiva e terapêutica.

\section{REFERÊNCIAS}

AGRA, K. O. A. et al. A Terapia Aquática como Coadjuvante na Variação do Humor em Mulheres Pós-Menopáusicas. Revista Brasileira de Ciências da Saúde, v. 17, n. 4, p. 327-334, 2013.

ALMEIDA, M, J, S. et al. Terapia cognitivo-comportamental em grupo para a disfunção sexual na pós-menopausa. J. Bras. Psiquiatr. Rio de Janeiro ,v. 67, n. 4, out./dez. 2018.

BARREeto, J, M.; OLIVEIRA, E, A, D. A Terapia Aquática como Coadjuvante na Variação do Humor em Mulheres Pós-Menopáusicas. Revista Brasileira de Ciências da Saúde. v.17, n. 4. p 327-334, 2013.

BAQUEDANO, V, M, M.; LARA, M, V. Disfunção sexual da mulher na vida reprodutiva. Rev. Cient. Esc. Univ. Cienc. Salud, 2017. 
BERBAM, LAURA WUTTIG. Exercícios de kegel e ginástica hipopressiva como estratégia de atendimento domiciliar no tratamento da incontinência urinária feminina: relato de caso. Revista Brasileira da Saúde, v.9, n. 6, 2012.

BERLEZI,E, M. et al. Incontinência urinária em mulheres no período pós menopausa: um problema de saúde pública. Rev. Bras. Geriatr. Gerontol., v. 12, n. 2, p. 159-173, 2009.

CARRIE, W, K,S .F. Efeitos da bola suiça para fortalecimento dos músculos do assoalho pélvico. International Journal of Cardiovascular Sciences, v.30, n 3, p. 227234, 2017.

CREMA, I, L., TILIO, R.; CAMPOS, M, P, A. Efeitos da menopausa na sexualidade feminina, Psicol. Cienc, v.37, n 3, Brasília, 2017.

FONSECA, E, J, N, et al. Síndrome Metabólica e Resistência Insulínica pelo Homa-IR no Climatério., Int. J. Cardiovasc. Sci, v.31 no.3, Rio de Janeiro maio/jun. 2018.

GALATI, M, C, R; DELMASCHIO, A, C, C; HORTA, A, L ,M Sexualidade de vida em mulheres com dificuldades sexuais. Psico-USF v.19, n.2, Itatiba maio/ago, 2014.

HOLANDA, J, B, L et al. Disfunção sexual e fatores associados relatados no período pós-parto. Acta paul. enferm. v. 27 n. 6, São Paulo nov./dez. 2014.

JESUS, J, C et al. A influência da ordem dos exercícios sobre o desempenho da força muscular em mulheres na pós-menopausa. MedicalExpress (São Paulo, online) v.5 São Paulo, 2018.

KAMILOS, M, F et al. Nova opção terapêutica na síndrome geniturinária da menopausa: estudo piloto utilizando radiofrequência fracionada microablativa.. Jornal Einstein, São Paulo, SP, Brasil, 2017.

LIMA, M, M, O et al. Exercício aeróbico no controle da hipertensão arterial na pósmenopausa. Fisioter. mov. (Impr.) v. 24, n.1 Curitiba Jan./Mar. 2011. 
LOPES, N, H, M et al. A Interface entre Osteoporose e Aterosclerose em Mulheres Pós-Menopausa,. Arq. Bras. Cardiol. v.110, n.3 São Paulo mar. 2018.

MARCONI, M. A.; LAKATOS, E. M. Técnicas de pesquisa: planejamento e execução de pesquisas, amostragens e técnicas de pesquisas, elaboração, análise e interpretação de dados. 5ed. São Paulo: Atlas, 2002.

MENDES, K, D, S; SILVEIRA, R, C, D, C, P; GALVÃO, C, M. Mmétodo de pesquisa para a incorporação de evidências na saúde. KEYWORDS: Research. Nursing. Health. Enferm, Florianópolis, Out-Dez; v. 17, n4. P 758-64, 2018

MENDONÇA, C. R.; AMARAL, W. N. Tratamento fisioterapêutico das disfunções sexuais femininas. Ver. Saúde da Mulher, v. 39, n. 3, p. 139-142, 2011.

PIOVESAN, A. TEMPORINI, E, R. Pesquisa exploratória: procedimento metodológico para o estudo de fatores humanos no campo da saúde pública. Rev. Saúde Pública v.29 n.4 São Paulo ago. 1995.

PIASSAROLLI, V. P. et al. Treinamento dos músculos do assoalho pélvico nas disfunções sexuais femininas. Rev Bras Ginecol Obstet., v. 32, n. 5, p. 234-40, 2010.

RIBEIRO, A, T, A; ANKEIER, C. A elação do grau de força muscular do assoalho pélvico com a satisfação sexual feminina. Revista Latino Americana de Medicina Sexual - Relams, vol.01, n. 01, 2012.

RUFINO, L; JUNIOR, I, F, F. EDUCAÇÃO SEXUAL NA SAÚDE GLOBAL. Rev. bras. cineantropom. desempenho humano v.18, n.1, Florianópolis jan./fev. 2013

SANTOS, A, M; SANTOS, F, C; CENDOROGLO, M, S. Sexualidade e dor crônica em idosas longevas: descrição de fatores interferências. Rev. Dor, v, 16, n.1, São Paulo jan./mar. 2015.

SIQUEIRA, J, B, D et al. Eficácia do tratamento fisioterapêutico na incontinência urinária em mulheres. Revista eletrônica saúde e ciência, v.07, n. 1, 2012. 
SILVA, V, H.; ROCHA, J, S ,B ; CALDEIRA, A, P. Fatores associados à autopercepção negativa de saúde em mulheres climatéricas., Ciência \& Saúde Coletiva v.23, n.5, p. 1611-1620, 2018.

SOUSA, J, C; FERREIRA, V, R.; OLIVEIRA, R, J; CESTARI, C, E. Avaliação da força muscular do assoalho pélvico em idosas com incontinência urinária. Fisioter. mov. (Impr.) v.24, n.1, Curitiba Jan./Mar.2010.

SOUZAM ,T, D; SILVA, M, D, D; CARVALHO, D, R. Revisão integrativa: o que é e como fazer. Einstein. V.8, n.1, 2013.

SOUZA, D, D, C, D et al. Efeitos de um Programa de Pilates sobre o equilíbrio de suas praticantes no período pós-menopaus. Rev. Kairós, v. 16, n.1, p. 39-49, mar. 2013.

STEIN, S, R.; PAVAN, F, V; NUNES, E, F, C; LATORRE, G, F, S. Entendimento da fisioterapia pélvica como opção de tratamento para as disfunções do assoalho pélvico por profissionais de saúde da rede pública. Rev. Ciênc. Méd. v.27, n. 2, p.65-72, 2018.

TOMEN, A.; FRACARO, G.; NUNES, E ,F, C; LATORRE, G, F, S. A fisioterapia pélvica no tratamento de mulheres portadoras de vaginismo. Rev. Ciênc. Méd., Campinas, v.24, n.3, p. 121-130, set./dez., 2015.

OLIVEIRA, E. L; NEVES, A. L. M.; SILVA, I. R.. Sentidos de sexualidade entre mulheres idosas: relações de gênero, ideologias mecanicistas e subversão,. Psicologia \& Sociedade, v. 30, n.16, 2018.

VALENTE, M, G et al. Efeitos da ginástica abdominal hipopressiva sobre a musculatura pélvica em mulheres incontinentes. Revista do Departamento de Educação Física e Saúde, Ano 16 - V. 16, n. 4, Outubro/De

Enviado: Junho, 2020.

Aprovado: Agosto, 2020. 